\title{
The dynamics of a central spin in quantum Heisenberg $X Y$ model
}

\author{
X.-Z. Yuan ${ }^{1, a}$, K.-D. Zhu ${ }^{1}$, and H.-S. Goan ${ }^{2}$ \\ 1 Department of Physics, Shanghai Jiao Tong University, Shanghai 200240, P.R. China \\ 2 Department of Physics and Center for Theoretical Sciences, National Taiwan University, Taipei 10617, Taiwan
}

Received 19 March 2007 / Received in final form 30 August 2007

Published online 24 October 2007 - (c) EDP Sciences, Società Italiana di Fisica, Springer-Verlag 2007

\begin{abstract}
In the thermodynamic limit, we present an exact calculation of the time dynamics of a central spin coupling with its environment at finite temperatures. The interactions belong to the Heisenberg $X Y$ type. The case of an environment with finite number of spins is also discussed. To get the reduced density matrix, we use a novel operator technique which is mathematically simple and physically clear, and allows us to treat systems and environments that could all be strongly coupled mutually and internally. The expectation value of the central spin and the von Neumann entropy are obtained.
\end{abstract}

PACS. 75.10.Jm Quantized spin models - 03.65.Yz Decoherence; open systems; quantum statistical methods - 03.67.-a Quantum information

\section{Introduction}

One of the promising candidates for quantum computation is spin systems [1-4] due to their long decoherence and relaxation time. Combined with nanostructure technology, they have the advantage of being scalable. Just as other quantum systems, the spin systems are inevitably influenced by their environment, especially the spin environment. As a result, decoherence will cause the transition of a system from pure quantum states to mixture classical ones. This is a big obstacle in the development of the quantum computer. Therefore the dynamic behavior of a single spin or several spins interacting with a spin bath has attracted much attention in recent years [5-13]. A quantum system exposed to environmental modes is described by the reduced density matrix. However, in most cases it is impossible to obtain an exact solution to the evolution of the reduced density matrix with the environmental modes traced over. Then different approximate methods are used. Markovian-type approximations [14-17] are valid only for relatively long times and fail to represent behavior of the system for short time regime [18]. On the other hand, some authors [18-20] develop short-time approximation to deal with the problem. If we can construct a physically relevant model which can be solved exactly, this will be a quite important work. Using a novel operator technique, we have studied the entanglement evolution of two coupled spins in a quantum spin environment in the thermodynamic limit at finite temperature [21]. In the present paper, we consider a similar model. However, to provide a complete picture of the dynamics, we study an observable

\footnotetext{
a e-mail: yxz@sjtu.edu.cn
}

like $S_{0}^{z}$ and extend the operator technique to deal with the case of finite number environmental spins. Recently, using numerical tools, the authors of references [5-8] studied the dynamic behavior of a central spin system decoherenced by a spin bath. They considered a general and realistic model. However the influence of environmental temperature was not taken into consideration. Here our model just involves the Heisenberg $X Y$ coupling which has extensive applications for various quantum information processing proposals [22-26]. Another benefit of this model is that we can obtain exactly the time evolution of the expectation value of central spin $S_{0}^{z}$ and the von Neumann entropy at finite temperature. Also the special operator technique we used is mathematically simple and physically clear, and allows us to treat systems and environments that could be strongly coupled mutually. Furthermore, the internal dynamics of the spin bath are taken into consideration. This is the main difference between our model and that in reference [27] where the system-bath coupling is also $X Y$ type, but the bath is assumed to be in an unpolarized infinite temperature states.

The paper is organized as follows. Section 2 introduces the model Hamiltonian. From the reduced density matrix, the expectation value of $S_{0}^{z}$ and the von Neumann entropy of the central spin are calculated. Conclusions are given in Section 3.

\section{Model and calculations}

We consider a single central spin (system) coupling with the bath spins via a Heisenberg $X Y$ interaction. The bath 
correlations are also $X Y$ type. Both the central spin and the bath consist of spin- $\frac{1}{2}$ atoms. $H_{B}$ is the Hamiltonian of the bath, and $H_{S B}$ represents the interaction between the central spin and the bath [27]

$$
H=H_{S B}+H_{B},
$$

where

$$
\begin{aligned}
H_{S B} & =\frac{g_{0}}{\sqrt{N}}\left(S_{0}^{+} \sum_{i=1}^{N} S_{i}^{-}+S_{0}^{-} \sum_{i=1}^{N} S_{i}^{+}\right), \\
H_{B} & =\frac{g}{N} \sum_{i \neq j}^{N}\left(S_{i}^{+} S_{j}^{-}+S_{i}^{-} S_{j}^{+}\right),
\end{aligned}
$$

here $S_{0}^{+}$and $S_{0}^{-}$are the spin-flip operators of the central spin, respectively. $S_{i}^{+}$and $S_{i}^{-}$are the corresponding operators of the $i$ th atom in the bath. $N$ is the number of the bath atoms which have direct interaction with the central spin. $g_{0}$ is the coupling constant between the central spin and the bath, whereas $g$ is that in the bath. Both constants are rescaled as $g_{0} / \sqrt{N}$ and $g / N$ [27-30]. Using the collective angular momentum operators $J_{ \pm}=\sum_{i=1}^{N} S_{i}^{ \pm}$, we rewrite the Hamiltonians as

$$
\begin{aligned}
H_{S B} & =\frac{g_{0}}{\sqrt{N}}\left(S_{0}^{+} J_{-}+S_{0}^{-} J_{+}\right), \\
H_{B} & =\frac{g}{N}\left(J_{+} J_{-}+J_{-} J_{+}\right)-g .
\end{aligned}
$$

After the Holstein-Primakoff transformation:

$$
J_{+}=\sqrt{N} b^{+}\left(1-\frac{b^{+} b}{N}\right)^{1 / 2}, J_{-}=\sqrt{N}\left(1-\frac{b^{+} b}{N}\right)^{1 / 2} b
$$

$b^{+}$and $b$ being creation and annihilation bosonic operators such that $\left[b, b^{+}\right]=1$, the Hamiltonians are written as

$$
\begin{aligned}
H_{S B} & =g_{0} S_{0}^{+}\left(1-\frac{b^{+} b}{2 N}\right) b+g_{0} S_{0}^{-} b^{+}\left(1-\frac{b^{+} b}{2 N}\right), \\
H_{B} & =2 g b^{+} b\left(1-\frac{b^{+} b}{N}\right) .
\end{aligned}
$$

We have made a $\frac{1}{N}$-expansion which is valid if the number of the atoms in excited states is much smaller than $N$ [28]. In the following, we can obtain exactly the reduced density matrix for the central spin by tracing over the bosonic bath at finite temperature.

We assume the initial density matrix of the composed system to be factorized, i.e., $\rho(0)=|\psi\rangle\langle\psi| \otimes \rho_{B}$. The initial state of the system is

$$
\begin{gathered}
|\psi\rangle=\alpha|1\rangle+\beta|0\rangle, \\
|\alpha|^{2}+|\beta|^{2}=1 .
\end{gathered}
$$

The density matrix of the bath satisfies the Boltzmann distribution, that is $\rho_{B}=e^{-H_{B} / T} / Z$, where $Z$ is the partition function and the Boltzmann constant has been set to one. At absolute zero temperature, no excitation will exist. The bath is in a thoroughly polarized state with all spin down. With the increase of temperature, the number of spin up atoms increases. $\rho_{S}(t)$ is the reduced density matrix of the central spin system, it can be written as

$$
\rho_{S}(t)=\operatorname{tr}_{B}\left\{e^{-i H t}\left[|\psi\rangle\langle\psi| \otimes \rho_{B}\right] e^{i H t}\right\},
$$

where $\operatorname{tr}_{B}$ denotes the partial trace taken over the Hilbert space of the spin bath. Using a novel operator technique, we can exactly determine the matrix $\rho_{S}(t)$ which is a $2 \times 2$ matrix in the standard basis $|0\rangle,|1\rangle$. As an example, we just calculate time evolution of the operator $|1\rangle\langle 1|$, i.e.,

$$
E(t)=\operatorname{tr}_{B}\left\{e^{-i H t}\left[|1\rangle\langle 1| \otimes e^{-H_{B} / T}\right] e^{i H t}\right\} .
$$

It is demonstrated in Appendix.

From equations (9), (11), and results in Appendix, the reduced density matrix can be written as

$$
\begin{aligned}
\rho_{S}(t) & =\operatorname{tr}_{B}\left\{e^{-i H t}\left[|\psi\rangle\langle\psi| \otimes \rho_{B}\right] e^{i H t}\right\} \\
& =\left(\begin{array}{ll}
\rho_{11} & \rho_{12} \\
\rho_{21} & \rho_{22}
\end{array}\right)
\end{aligned}
$$

where

$$
\begin{aligned}
\rho_{11}= & |\alpha|^{2} \frac{1}{Z} \sum_{n=0}^{M} A_{1} A_{1}^{*} e^{-2 g n\left(1-\frac{n}{N}\right) / T} \\
& +|\beta|^{2} \frac{1}{Z} \sum_{n=1}^{M} C_{1} C_{1}^{*} n e^{-2 g n\left(1-\frac{n}{N}\right) / T}, \\
\rho_{12}= & \alpha \beta^{*} e^{-i 2 g t} \frac{1}{Z} \sum_{n=0}^{M} A_{1} D_{1}^{*} e^{-2 g n\left(1-\frac{n}{N}\right) / T}, \\
\rho_{21}= & \alpha^{*} \beta e^{i 2 g t} \frac{1}{Z} \sum_{n=0}^{M} D_{1} A_{1}^{*} e^{-2 g n\left(1-\frac{n}{N}\right) / T}, \\
\rho_{22}= & |\alpha|^{2} \frac{1}{Z} \sum_{n=0}^{M} B_{1} B_{1}^{*}(n+1) e^{-2 g n\left(1-\frac{n}{N}\right) / T} \\
& +|\beta|^{2} \frac{1}{Z} \sum_{n=0}^{M} D_{1} D_{1}^{*} e^{-2 g n\left(1-\frac{n}{N}\right) / T} .
\end{aligned}
$$

As mentioned in Appendix, the operator $\hat{n}$ has been replaced by its eigenvalue $n$.

Assuming an initial condition $\alpha=1$ and $\beta=0$, we obtain the expectation value of $S_{0}^{z}$

$$
\begin{aligned}
\left\langle S_{0}^{z}\right\rangle & =\operatorname{tr}_{S}\left(S_{0}^{z} \rho_{S}\right) \\
& =\left\langle 1\left|S_{0}^{z} \rho_{S}\right| 1\right\rangle+\left\langle 0\left|S_{0}^{z} \rho_{S}\right| 0\right\rangle \\
& =\frac{1}{2 Z} \sum_{n=0}^{M}\left[A_{1} A_{1}^{*}-B_{1} B_{1}^{*}(n+1)\right] e^{-2 g n\left(1-\frac{n}{N}\right) / T},
\end{aligned}
$$

where $\operatorname{tr}_{S}$ denotes the partial trace taken over the Hilbert space of the central spin. 


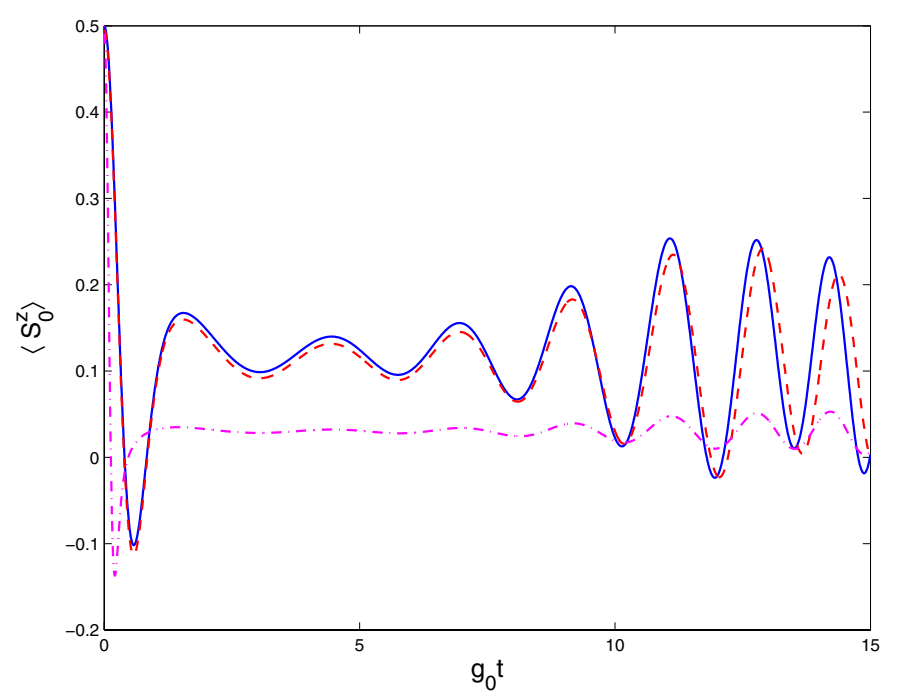

Fig. 1. Time evolution of $\left\langle S_{0}^{z}\right\rangle$ for an initial system state $|\psi\rangle=$ $|1\rangle$ for different temperatures. $g=g_{0}, T=100 g$ (dot dashed curve), $T=10 g$ (solid curve), and $T=10 g, N=280$ (dashed curve).

In Figure 1, we plot $\left\langle S_{0}^{z}\right\rangle$ as a function of time for different values of temperature $T$. Except the dot dash curve which is for $T=10 \mathrm{~g}$ and $N=280$, all the results below are in the thermodynamic limit. It shows that initially $\left\langle S_{0}^{z}\right\rangle$ exhibits a coherent oscillatory decay. With the increase of temperature, the decay rate increases. After a turning point $g_{0} t \approx 6$, which is independent on the temperature, the oscillatory amplitude increases. Then $\left\langle S_{0}^{z}\right\rangle$ oscillates in certain low value regime at high temperature. After the initial fast decay, the central system exhibits long-time oscillation. This is quite an unexpected result. It is a common sense that interaction of the system with its environment leads to a decay of the system's initial pure state into a mixture of several states, i.e., non diagonal elements of the density matrix vanish, and diagonal elements achieve their equilibrium values. This causes an damping of quantum oscillations and increase of the system's entropy with time [5]. However our results are not unique. Recently, the authors of references [5,6] studied numerically the dynamic behavior of a two spin system decoherenced by a spin bath. Without considering the influence of the environmental temperature, they used a general and realistic model and found that the system shows a special dynamic behavior, i.e., an initial fast decay of $\left\langle S_{1}^{z}\right\rangle$ or $\left\langle S_{2}^{z}\right\rangle$ is followed by revival oscillations. This new phenomenon was explained in detail, where the crucial factor is whether the central system comprises even or odd number of spin entities $[5,6]$. Here we consider a single central spin coupling with a spin bath. All the interactions belong to the $X Y$ type. At finite temperature, an exact solution is given. We do not think the mechanism of the revival oscillation in our paper is same as that in references [5,6]. Due to the symmetry of our model, the Hamiltonian after the Holstein-Primakoff transformation and in the thermodynamic limit is equivalent to that of a qubit interacting with a single-mode thermal bosonic field.
The effect of this single-mode environment on the dynamics of the central qubit is extremely non-Markovian. The quantum information flowing into the environment may partially return to the central spin. This reflects onto, for example, the revival behavior of the reduced density matrix of the central spin. This is different from the usual environment model which consists of many bosonic modes and often causes the reduced dynamics of the system of interest displaying an exponential decay in time behavior. So the Markovian approximation usually used in quantum optics master equation will not work in our model. One may do perturbation theory for weak-coupling case, but the single-mode environment in our model will not remain in thermal equilibrium state as is usually assumed for an environment with very large degrees of freedom in the weak-coupling master equation approach. As our model corresponds to an extremely non-Markovian case, the dynamics of $\left\langle S_{0}^{z}\right\rangle$ shows a two-step behavior. That is, after an initially quicker decay of the amplitude, the dynamics still shows a behavior of quantum oscillations at long times. The general trend for temperature dependent dynamics is that the larger the temperature is, the quicker the initial decay is and the smaller the amplitude of the long-time oscillations is. This can be explained as follows. At high temperature, the environment appears to be in very disorderly states. The central spin quickly loses its message to the environment and only a little message can return to it. At infinite high temperature, no message will return to the central spin. Then the revival behavior will disappear. This is the case of reference [27], where the spin bath is assumed to be in an unpolarized infinite temperature state. In practice, only finite number of spins in the bath interact with the central spin. Therefore it is necessary to investigate the working condition of the thermodynamic limit in our model. The good fitness between solid curve and dashed curve in Figure 1 shows that it is safe to use the thermodynamic limit if $T<10 \mathrm{~g}$ and $N>280$.

The von Neumann entropy of the central spin is defined as

$$
P=-\operatorname{tr}_{B}\left(\rho_{S} \ln \rho_{S}\right)
$$

After diagonalising the reduced density matrix $\rho_{S}$, we obtain the von Neumann entropy

$$
P=-\sum_{k=1}^{2} p_{k} \ln p_{k},
$$

where $p_{k}$ are the eigenvalues of the reduced density matrix $\rho_{S}$ and can be expressed as

$$
p_{1,2}=\frac{\rho_{11}+\rho_{22} \pm \sqrt{\left(\rho_{11}-\rho_{22}\right)^{2}+4 \rho_{12} \rho_{21}}}{2} .
$$

In Figure 2, we plot the von Neumann entropy $P(t)$ of the reduced system for different values of $T$. The initial state of the system is

$$
|\psi\rangle=\frac{\sqrt{2}}{2}|1\rangle+\frac{\sqrt{2}}{2}|0\rangle .
$$




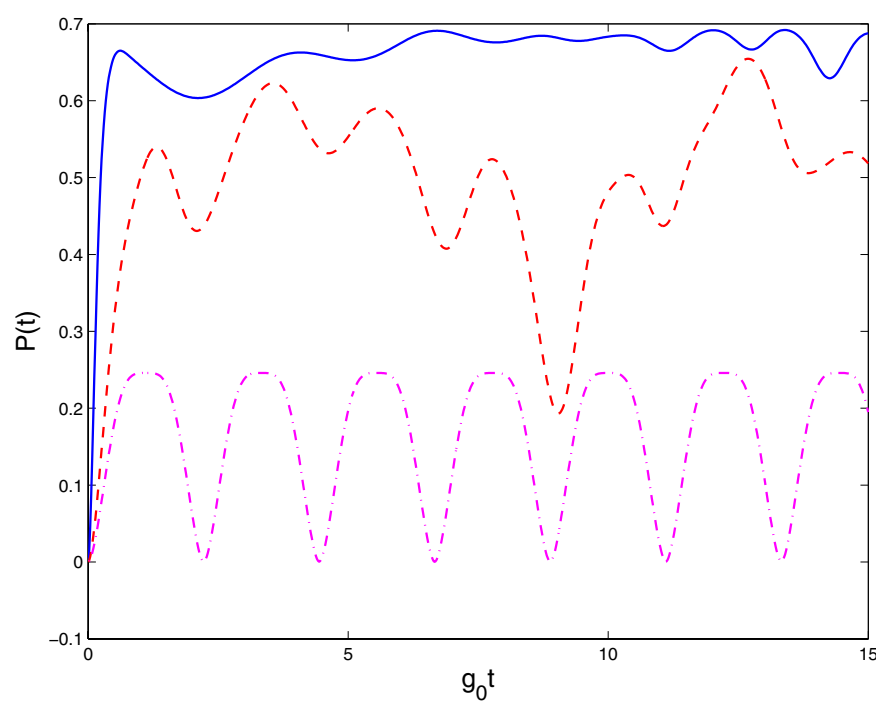

Fig. 2. Time evolution of entropy $P(t)$ for an initial system state $|\psi\rangle=\frac{\sqrt{2}}{2}|1\rangle+\frac{\sqrt{2}}{2}|0\rangle$ for different temperatures. $g=g_{0}$, $T=20 g$ (solid curve), $T=2 g$ (dashed curve), and $T=0.2 g$ (dot dashed curve).

At high temperature, our results agree with reference [27] quite well, especially for the initial stage of evolution, where the Von Neumann entropy increases quickly. However, some kinds of oscillations appear at long times. This shows the Non-Markovian behavior of the central spin system. That is the system exchanges entropy (quantum information) with the spin bath for a long time at finite temperature, so that the entropy will not come close to saturation. With the decrease of temperature, the increasing rate of $P(t)$ becomes smaller and $P(t)$ oscillates in a lower value regime. For $T=2 g$, we see a large amplitude vibration which means that non-equilibrium properties of the system become more obvious. At very low temperature, the system still exchange entropy with the spin bath periodically. In such case, the bath is in a state with all spin down. The initial state of the central spin contains spin up component. The interaction is $X Y$ type, then the central spin and the spin bath can still exchange entropy at very low temperature. If the central spin is also in a spin down state, no exchange will happen.

\section{Conclusions}

We study the exact dynamics of a central spin in the quantum Heisenberg $X Y$ model in the thermodynamic limit. The reduced dynamics of the central spin coupling with its environment at finite temperature is obtained. From the reduced dynamics matrix, we calculate the expectation value of the central spin $S_{0}^{z}$. The results show that it decreases quickly and oscillates in a low value regime at high temperature. We also calculate the von Neumann entropy of the system. At very low temperature, with the initial pure state $|\psi\rangle=\frac{\sqrt{2}}{2}|1\rangle+\frac{\sqrt{2}}{2}|0\rangle$, the central spin evolves far from the completely mixed state $P_{\max }=\ln 2$.
X.Z.Y. acknowledges support from the National Natural Science Foundation of China under Grant No. 10647137. H.S.G. would like to acknowledge support from the National Science Council, Taiwan, under Grant No. 96-2112-M-002-007.

\section{Appendix: Calculation of time evolution of the operator $|1\rangle\langle 1|$}

The total Hamiltonian $H$ contains operators $b, b^{+}, S_{0}^{-}$, and $S_{0}^{+}$. Here $S_{0}^{-}$and $S_{0}^{+}$change the system state from $|1\rangle$ to $|0\rangle$, and vice versa. Since

$$
e^{-i H t}=1-i H t+\frac{(i H t)^{2}}{2 !}+\ldots,
$$

it is obvious that we can write

$$
e^{-i H t}|1\rangle|x\rangle=\gamma|1\rangle\left|x^{\prime}\right\rangle+\delta|0\rangle\left|x^{\prime \prime}\right\rangle,
$$

where $|x\rangle$ is an arbitrary reference state on the bath and $\gamma$ and $\delta$ are two time dependent numbers. Defining $A|x\rangle=$ $\gamma\left|x^{\prime}\right\rangle$ and $B|x\rangle=\delta\left|x^{\prime \prime}\right\rangle$, we have

$$
e^{-i H t}|1\rangle|x\rangle=(A|1\rangle+B|0\rangle)|x\rangle,
$$

where $A$ and $B$ are functions of operators $b, b^{+}$, and time $t$. Using the Schrödinger equation identity

$$
i \frac{d}{d t}\left(e^{-i H t}|1\rangle|x\rangle\right)=H\left(e^{-i H t}|1\rangle|x\rangle\right),
$$

and equation (A.3), we obtain

$$
\begin{aligned}
& \frac{d}{d t} A=-i\left[g_{0}\left(1-\frac{b^{+} b}{2 N}\right) b B+2 g b^{+} b\left(1-\frac{b^{+} b}{N}\right) A\right], \\
& \frac{d}{d t} B=-i\left[g_{0} b^{+}\left(1-\frac{b^{+} b}{2 N}\right) A+2 g b^{+} b\left(1-\frac{b^{+} b}{N}\right) B\right],
\end{aligned}
$$

with initial conditions from equation (A.3) being $A(0)=1$ and $B(0)=0$. As $A$ and $B$ are functions of $b^{+}$and $b$, they are operators and do not commute with each other. Equations (A.5) and (A.6) are thus coupled differential equations of non-commuting operator variables, which can not be solved by using conventional methods for ordinary number variables.

The crucial observation to solve the problem is that the Hamiltonian, equations (7) and (8), is of an effective Jaynes-Cumming type and it can be block-diagonalized in the dressed state subspace of $|i ; n\rangle$, with $i+n=$ constant. Here $|i\rangle$ represent the qubit states and $|n\rangle$ are the bosonic field number states. As a result, we may rewrite equations (A.5) and (A.6) in such a subspace. By introducing the following transformation

$$
\begin{aligned}
& A=A_{1}, \\
& B=b^{+} B_{1},
\end{aligned}
$$


equations (A.5) and (A.6) then become

$$
\begin{aligned}
& \frac{d}{d t} A_{1}=-i\left[g_{0}(\hat{n}+1)\left(1-\frac{\hat{n}}{2 N}\right) B_{1}+2 g \hat{n}\left(1-\frac{\hat{n}}{N}\right) A_{1}\right], \\
& \frac{d}{d t} B_{1}=-i\left[g_{0}\left(1-\frac{\hat{n}}{2 N}\right) A_{1}+2 g(\hat{n}+1)\left(1-\frac{\hat{n}+1}{N}\right) B_{1}\right],
\end{aligned}
$$

where $\hat{n}=b^{+} b$. At this stage, we know that $A_{1}, B_{1}$ are functions of $\hat{n}$ and $t$, and commute with each other. We can then treat equations (A.9) and (A.10) as coupled complexnumber differential equations and solve them in a usual way. Considering initial condition

$$
\begin{aligned}
& A_{1}(0)=1, \\
& B_{1}(0)=0,
\end{aligned}
$$

we obtain

$$
\begin{aligned}
A_{1}= & \frac{2 g(\hat{n}+1)\left(1-\frac{\hat{n}+1}{N}\right)+\lambda_{1}}{2 \sqrt{g^{2}\left(1-\frac{2 \hat{n}+1}{N}\right)^{2}+g_{0}^{2}(\hat{n}+1)\left(1-\frac{\hat{n}}{2 N}\right)^{2}}} e^{i \lambda_{1} t} \\
& -\frac{2 g(\hat{n}+1)\left(1-\frac{\hat{n}+1}{N}\right)+\lambda_{2}}{2 \sqrt{g^{2}\left(1-\frac{2 \hat{n}+1}{N}\right)^{2}+g_{0}^{2}(\hat{n}+1)\left(1-\frac{\hat{n}}{2 N}\right)^{2}}} e^{i \lambda_{2} t}, \\
B_{1}= & -\frac{g_{0}\left(1-\frac{\hat{n}}{2 N}\right)}{2 \sqrt{g^{2}\left(1-\frac{2 \hat{n}+1}{N}\right)^{2}+g_{0}^{2}(\hat{n}+1)\left(1-\frac{\hat{n}}{2 N}\right)^{2}}} \\
& \times\left[e^{\left.i \lambda_{1} t-e^{i \lambda_{2} t}\right],}\right.
\end{aligned}
$$

where

$$
\begin{aligned}
\lambda_{1,2}= & -g(2 \hat{n}+1)+g \frac{2 \hat{n}^{2}+2 \hat{n}+1}{N} \\
& \pm \sqrt{g^{2}\left(1-\frac{2 \hat{n}+1}{N}\right)^{2}+g_{0}^{2}(\hat{n}+1)\left(1-\frac{\hat{n}}{2 N}\right)^{2}} .
\end{aligned}
$$

Then finally we have

$$
\begin{aligned}
E(t)= & |1\rangle\langle 1| \frac{1}{Z} \sum_{n=0}^{M} A_{1} A_{1}^{*} e^{-2 g n\left(1-\frac{n}{N}\right) / T} \\
& +|0\rangle\langle 0| \frac{1}{Z} \sum_{n=0}^{M} B_{1} B_{1}^{*}(n+1) e^{-2 g n\left(1-\frac{n}{N}\right) / T},
\end{aligned}
$$

where

$$
Z=\sum_{n=0}^{M} e^{-2 g n\left(1-\frac{n}{N}\right) / T} .
$$

In equation (A.16), the trace over the environmental degrees of freedom has been performed and the operator $\hat{n}$ has been replaced by its eigenvalue $n$. We have made a $\frac{1}{N}$-expansion in equations (7) and (8), which is valid only for $n \ll N$. Therefore we demand $M \ll N$ for the integer $M$ in equation (A.16). Also equation (A.16) must converge quickly for $M \ll N$, which is the case at low temperature $(T \ll g)$. In the thermodynamic limit $(N \rightarrow \infty)$, we can set $M \rightarrow \infty$ and discard the limitation of the low temperature. In such case, the partition function

$$
Z=\frac{1}{1-e^{-2 g / T}}
$$

Let

$$
e^{-i H t}|0\rangle|x\rangle=(C|1\rangle+D|0\rangle)|x\rangle .
$$

In the same way, we have

$$
\begin{aligned}
& C=b C_{1} e^{i 2 g t}, \\
& D=D_{1} e^{i 2 g t},
\end{aligned}
$$

where

$$
\begin{aligned}
C_{1}= & -\frac{g_{0}\left(1-\frac{\hat{n}-1}{2 N}\right)}{2 \sqrt{g^{2}\left(1-\frac{2 \hat{n}-1}{N}\right)^{2}+g_{0}^{2} \hat{n}\left(1-\frac{\hat{n}-1}{2 N}\right)^{2}}} \\
& \times\left[e^{i \lambda_{1}^{\prime} t}-e^{i \lambda_{2}^{\prime} t}\right] \\
D_{1}= & \frac{2 g \hat{n}-2 g \frac{(\hat{n}-1)^{2}}{N}+\lambda_{1}^{\prime}}{2 \sqrt{g^{2}\left(1-\frac{2 \hat{n}-1}{N}\right)^{2}+g_{0}^{2} \hat{n}\left(1-\frac{\hat{n}-1}{2 N}\right)^{2}}} e^{i \lambda_{1}^{\prime} t} \\
& -\frac{2 g \hat{n}-2 g \frac{(\hat{n}-1)^{2}}{N}+\lambda_{2}^{\prime}}{2 \sqrt{g^{2}\left(1-\frac{2 \hat{n}-1}{N}\right)^{2}+g_{0}^{2} \hat{n}\left(1-\frac{\hat{n}-1}{2 N}\right)^{2}}} e^{i \lambda_{2}^{\prime} t},
\end{aligned}
$$

where

$$
\begin{aligned}
\lambda_{1,2}^{\prime} & =-g(2 \hat{n}+1)+g \frac{2 \hat{n}^{2}-2 \hat{n}+1}{N} \\
& \pm \sqrt{g^{2}\left(1-\frac{2 \hat{n}-1}{N}\right)^{2}+g_{0}^{2} \hat{n}\left(1-\frac{\hat{n}-1}{2 N}\right)^{2}} .
\end{aligned}
$$

\section{References}

1. D. Loss, D.P. Divincenzo, Phys. Rev. A 57, 120 (1998)

2. G. Burkard, D. Loss, D.P. Divincenzo, Phys. Rev. B 59, 2070 (1999)

3. A. Imamoglu, D.D. Awschalom, G. Burkard, D.P. DiVincenzo, D. Loss, M. Sherwin, A. Small, Phys. Rev. Lett. 83, 4204 (1999)

4. R. Raussendorf, H.J. Briegel, Phys. Rev. Lett. 86, 5188 (2001)

5. V.V. Dobrovitski, H.A. De Raedt, M.I. Katsnelson, B.N. Harmon, Phys. Rev. Lett. 90, 210401 (2003)

6. V.V. Dobrovitski, H.A. De Raedt, Phys. Rev. E 67, 056702 (2003)

7. J. Lages, V.V. Dobrovitski, M.I. Katsnelson, H.A. De Raedt, B.N. Harmon, Phys. Rev. E 72, 026225 (2005)

8. W.X. Zhang, V.V. Dobrovitski, K.A. Al-Hassanieh, E. Dagotto, B.N. Harmon, Phys. Rev. B 74, 205313 (2006)

9. F.M. Cucchietti, J.P. Paz, W.H. Zurek, Phys. Rev. A 72, $052113(2005)$ 
10. W.H. Zurek, Rev. Mod. Phys. 75, 715 (2003)

11. N.V. Prokof'er, P.C.E. Stamp, Rep. Prog. Phys. 63, 669 (2000)

12. S. Camalet, R. Chitra, Phys. Rev. B 75, 094434 (2007)

13. J. Schliemann, A.V. Khaetskii, D. Loss, Phys. Rev. B 66, 245303 (2002)

14. W.H. Louisell, Quantum Statistical properties of Radiation (Wiley, 1973)

15. K. Blum, Density Matrix Theory and Applications (Plenum Press, NY, 1996)

16. H. Grabert, P. Schramm, G.-L. Ingold, Phys. Rep. 168, 115 (1988)

17. A.J. Berkley, H. Xu, M.A. Gubrud, R.C. Ramos, J.R. Anderson, C.J. Lobb, F.C. Wellstood, Phys. Rev. B 68, 060502(R) (2003)

18. V. Privman, Mod. Phys. Lett. B 16, 459 (2002)

19. V. Privman, J. Stat. Phys. 110, 957 (2003)

20. D. Tolknuov, V. Privman, Phys. Rev. A 69, 062309 (2004)
21. X.Z. Yuan, H.S. Goan, K.D. Zhu, Phys. Rev. B 75, 045331 (2007)

22. D.A. Lidar, L.A. Wu, Phys. Rev. Lett. 88, 017905 (2002)

23. L.A. Wu, D.A. Lidar, M. Friesen, Phys. Rev. Lett. 93, $030501(2004)$

24. C.D. Hill, H.-S. Goan, Phys. Rev. A 68, 012321 (2003)

25. A. Blais, R.-S. Huang, A. Wallraff, S.M. Girvin, R.J. Schoelkopf, Phys. Rev. A 69, 062320 (2004)

26. M. Sarovar, H.-S. Goan, T.P. Spiller, G.J. Milburn, Phys. Rev. A 72, 062327 (2005)

27. H.P. Breuer, D. Burgarth, F. Petruccione, Phys. Rev. B 70, 045323 (2004)

28. M. Frasca, Ann. Phys. 313, 26 (2004)

29. S. Paganelli, F. de Pasquale, S.M. Giampaolo, Phys. Rev. A 66, 052317 (2002)

30. M. Lucamarini, S. Paganelli, S. Mancini, Phys. Rev. A 69, $062308(2004)$ 